\title{
Intracerebral lipopolysaccharide induces neuroinflammatory change and augmented brain injury in growth-restricted neonatal rats
}

\author{
Leigh R. Campbell', Yi Pang ${ }^{1}$, Norma B. Ojeda', Baoying Zheng ${ }^{1}$, Philip G. Rhodes' ${ }^{1}$, and Barbara T. Alexander ${ }^{2}$
}

INTRODUCTION: Intrauterine growth restriction (IUGR) alters fetal development and is associated with neurodevelopmental abnormalities. We hypothesized that growth restriction from reduced intrauterine perfusion would predispose neonatal rats to subsequent inflammatory brain injury.

METHODS: In this study, IUGR was achieved by induced placental insufficiency in pregnant rats at 14 days of gestation. IUGR offspring and sham-operated control pups were subsequently injected with intracerebral lipopolysaccharide (LPS) as a model of periventricular leukomalacia (PVL).

RESULTS: LPS similarly elevates proinflammatory cytokines in the brains of both IUGR and control rat pups. However, the chemokines cytokine-induced neutrophil chemoattractant-1 (CINC-1) and macrophage chemoattractant protein-1 (MCP-1), as well as microglia activation, were significantly higher in LPS-treated IUGR rat pups as compared with LPS-treated controls. In addition to the unique brain inflammatory response, IUGR rat pups demonstrated increased brain damage with an increased number of apoptotic cells, larger lateral ventricular size, and more severe impairment of myelination.

DISCUSSION: This study provides evidence that placental insufficiency may sensitize the innate immune system in the immature brain and reveals a possible link between brain inflammation and injury.

\footnotetext{
ntrauterine growth restriction (IUGR) is failure of the fetus to achieve intrinsic growth potential, leading to increased morbidity and mortality (1). A disorder of the fetoplacentalmaternal unit is the major etiology of compromised intrauterine growth in developed societies (2). In asymmetric growth restriction, fetal blood flow is redistributed to the brain, resulting in an increase in brain mass relative to an overall reduction in body weight (3). There exists an apparent physical protection of the brain; however, in clinical practice IUGR infants demonstrate an increased risk for neurodevelopmental abnormalities, including intracranial injury, cerebral palsy, psychological disorders, cognitive defects, and behavior problems $(4,5)$. Chronic in utero stress and hypoxia associated with reduced intrauterine perfusion results in adaptive responses that can provide enhanced
}

protection or an increased vulnerability to future sublethal insults $(6,7)$. It remains to be elucidated whether the brains of IUGR newborns are preconditioned and subsequently more tolerant to severe insults experienced in the perinatal period, or sensitized and therefore more vulnerable (8). In our established model, intracerebral injection of lipopolysaccharide (LPS) causes an inflammatory response in rat brain similar to the pathology seen in periventricular leukomalacia (PVL; refs. 9-11). Clinically, hypoxia-ischemia and infection/inflammation often coexist and interact with each other. Therefore, we hypothesize that chronic in utero stress from reduced intrauterine perfusion sensitizes the immature rat brain to subsequent inflammatory stress. We demonstrate an exacerbated inflammatory response to LPS in the growth-restricted rat brain that is associated with significantly increased brain injury relative to appropriately grown rat pups.

\section{RESULTS}

\section{Brain Inflammatory Responses to LPS}

Cytokine array was used to identify differentially expressed cytokines/chemokines in the control and IUGR rats at both 6 and $24 \mathrm{~h}$ after LPS treatment. Major proinflammatory cytokines such as tumor necrosis factor- $\alpha$, interleukin-1 $\beta$ (IL-1 $\beta$ ), and IL- 6 were elevated in IUGR and control rat pups to the same degree. However, two chemokines, macrophage chemoattractant protein-1 (MCP-1) and cytokine-induced neutrophil chemoattractant-1 (CINC-1), as well as tissue inhibitor of metalloproteinase-1, were significantly higher in IUGR-LPS rat pups as compared with control-LPS rats at $24 \mathrm{~h}$ after LPS injection (Figure 1a). Enzyme-linked immunosorbent assay studies were conducted to evaluate for markers of inflammation as well as to confirm and quantify cytokine array results. An increase in expression of the proinflammatory cytokines tumor necrosis factor- $\alpha$ and IL- 6 was observed in the control and IUGR rats treated with LPS at both 6 and $24 \mathrm{~h}$ (data not shown). At $24 \mathrm{~h}$, the IL-1 $\beta$ level was lower in IUGR-LPS rats as compared with control-LPS rats (control-LPS: $706.8 \pm 92.3 \mathrm{pg} / \mathrm{mg}$ protein, $n=8$; IUGR-LPS: $523.6 \pm 38.9 \mathrm{pg} / \mathrm{mg}$ protein, $n=10$; mean $\pm \mathrm{SD}$ ); however, it did not reach statistical difference 


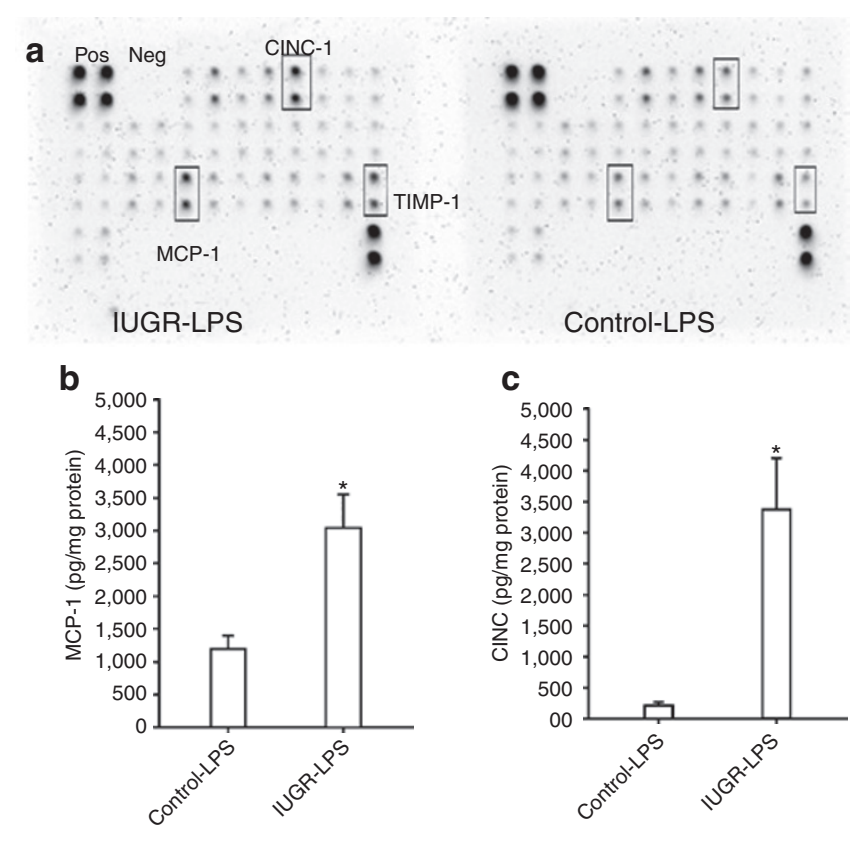

Figure 1. Inflammatory response in rat brain after LPS injection. (a) Cytokine antibody array was used as an initial step to screen and quantify differentially expressed cytokines/chemokines as described in the Methods section. Chemokines CINC-1 and MCP-1, as well as TIMP-1, were found to be differentially expressed (in duplicate, labeled in boxes). $(\mathbf{b}, \mathbf{c})$ ELISA was performed to further quantify major proinflammatory chemokines and cytokines. There were no significant differences in expression of acute-phase cytokines (TNF, IL-1, and IL-6) between the control-LPS and IUGR-LPS groups. However, consistent with array data, chemokines MCP-1 and CINC-1 were significantly higher in the IUGR-LPS than the control-LPS group. Proinflammatory cytokines MCP-1 and CINC-1 were undetectable in control and IUGR rat pups not treated with LPS. ${ }^{*} P<0.01$ vs. control-LPS. CINC-1, cytokine-induced neutrophil chemoattractant-1; ELISA, enzyme-linked immunosorbent assay; IL, interleukin; IUGR, intrauterine growth restriction; LPS, lipopolysaccharide; MCP-1, macrophage chemoattractant protein-1; Neg, negative; Pos, positive; TIMP-1, tissue inhibitor of metalloproteinase-1;TNF, tumor necrosis factor.

$(P=0.065)$. The levels of two chemokines, MCP-1 and CINC-1 (rat IL-8), were significantly higher in IUGR-LPS rats as compared with control-LPS rats at $24 \mathrm{~h}$. Specifically, the MCP- 1 level was about 2.5 times higher in IUGR-LPS rats relative to control-LPS rats, whereas the increase in CINC-1 level was $>16$ times greater (Figure $\mathbf{1 b}, \mathbf{c}$ ). Proinflammatory cytokines and chemokines were not detected in the brains of control or IUGR rats without LPS treatment.

To determine differential responses of the microglia and astrocytes between IUGR and control rats following LPS treatment, immunohistochemistry studies using an ED1 antibody (a marker for activated microglia) revealed that microglia were activated in both control and IUGR rat brains following LPS exposure. However, IUGR-LPS rats showed marked enhancement of microglia activation as compared with control-LPS rats at $24 \mathrm{~h}$ (Figure 2). Although a few $\mathrm{ED}^{+}$cells were present in the periventricular white matter tracts in the brains of both groups without LPS treatment, there was no difference noted between the two (data not shown). Following LPS injection, $\mathrm{ED1}^{+}$cells increased significantly in both groups; however, the IUGR group demonstrated more enhanced activation as compared with the control group. Moreover, the enhancement demonstrated a regional difference, with the enhancement in the striatum being more prominent (4.6-fold increase) than that in the periventricular white matter (1.6-fold increase; Figure $2 \mathrm{~g}, \mathrm{~h}$ ). Enhanced activation of microglia was consistently observed in IUGR-LPS rat brain over time at postnatal day 8 (P8) and P14 (data not shown).

In contrast to a marked increase of microglia activation, astrocyte activation appeared delayed in IUGR rat brain after LPS treatment. There were significantly fewer glial fibrillary acidic protein-positive $\left(\mathrm{GFAP}^{+}\right)$cells in IUGR-LPS than control-LPS brain at P6 and P8, but this difference was no longer apparent at P14 (Figure 3).

\section{Cell Death Following LPS Exposure}

Cell death in response to LPS was examined using the terminal deoxynucleotidyl transferase nick-end labeling (TUNEL) method. There were similar numbers of occasional scattered TUNEL $^{+}$cells in both groups without LPS treatment, but there appeared to be no difference between these two groups. Consistent with our previous observation, LPS treatment increased the number of TUNEL ${ }^{+}$cells in the control rat brain tissue, primarily in layer I of the cortex after $24 \mathrm{~h}$ of treatment. However, the increase of TUNEL ${ }^{+}$cells in the IUGR-LPS group more than doubled as compared with the control-LPS group $(P<0.001)$. In addition to the cortex, $\mathrm{TUNEL}^{+}$cells were found to be significantly increased in other brain areas in the IUGR-LPS rat group, most prominently in the striatum (Figure 4).

\section{LPS-Induced Lateral Ventricle Dilation}

A consistent gross pathological change in neonatal rat brain following LPS exposure is the dilation of the lateral ventricles $(12,13)$. As expected, the lateral ventricles were significantly enlarged in the control rat brain 9 days after exposure to LPS (Figure 5). This effect, however, was further exaggerated in IUGR-LPS rats $(P<0.05)$.

\section{Reduction of Myelination After LPS Treatment}

At P14, myelin is extensively detected in the white matter by myelin basic protein (MBP) immunostaining, and the control and IUGR rat brains showed similar intensity and pattern of immunostaining. After LPS exposure, there was a significant decline of MPB immunostaining in both groups as compared with their corresponding non-LPS treated groups. However, IUGR-LPS rat brains showed an enhanced reduction as compared with control-LPS rat brains $(P<0.05$; Figure 6$)$.

\section{DISCUSSION}

PVL is a prevalent form of brain injury in the premature infant that occurs following hypoxic-ischemic and inflammatory conditions (8). Utilizing an established model of neuroinflammation (10), we investigated whether IUGR enhances susceptibility to LPS insult. The major findings from our study indicate that IUGR rat pups produce an augmented inflammatory response and subsequent brain injury 

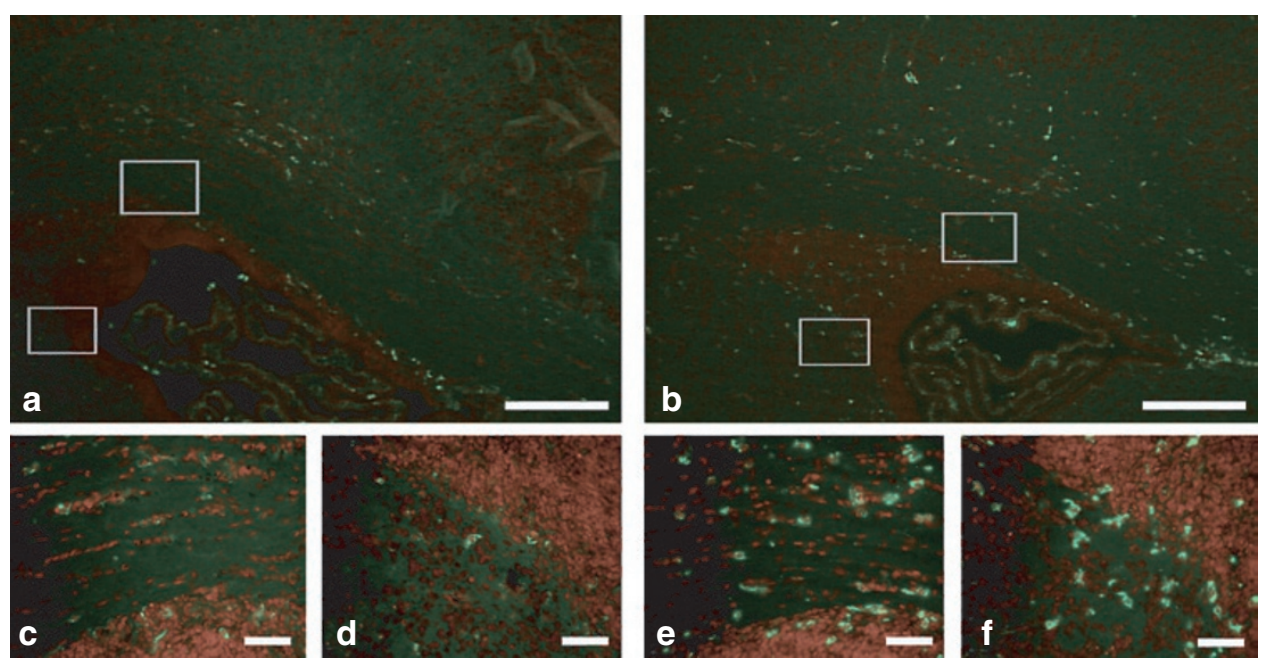

g

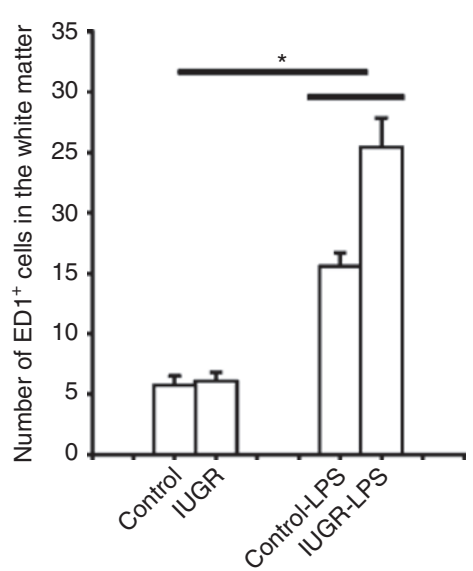

h

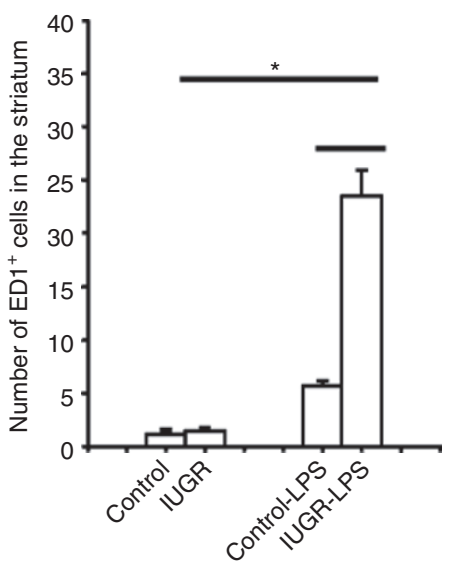

Figure 2. Microglia response in rat brain after LPS injection. A minimal number of ED1 ${ }^{+}$cells were detected in both control and IUGR rat brains without LPS exposure at P6. At $24 \mathrm{~h}$ following intracerebral LPS injection, the number of ED $1^{+}$cells increased significantly in the periventricular white matter of both the (a) control and (b) IUGR rat brain; however, this response was intensified in IUGR-LPS rat brains. The highlighted areas within the white boxes at the corpus callosum (upper) and the junction between the subventricular zone and striatum (lower) in a are shown in higher magnifications in $\mathbf{c}$ and $\mathbf{d}$, respectively, while those in $\mathbf{b}$ are shown as e and $\mathbf{f}$. Bar $=\mathbf{a}, \mathbf{b}: 200 \mu \mathrm{m} ; \mathbf{c}-\mathbf{f}: 50 \mu \mathrm{m}$. ED1 ${ }^{+}$cells were counted in the white matter and striatum at P6 and the data are shown in $\mathbf{g}$ and $\mathbf{h}$, respectively. ${ }^{*} P<0.01$, IUGR-LPS vs. control-LPS; control or IUGR with LPS vs. without LPS. IUGR, intrauterine growth restriction; LPS, lipopolysaccharide; $P$, postnatal day.

as compared with control rat pups. To our knowledge, this is the first study that suggests that placental insufficiency programs the infant brain to be more vulnerable to postnatal inflammatory challenges.

Consistent with previous studies $(12,13)$, we show similarly increased expression of proinflammatory cytokines following LPS exposure, but specific chemokines, including MCP-1 and CINC-1, were significantly elevated to a greater degree in growth-restricted rat pups. Activated macrophages/ microglia, seen in the pathogenesis of PVL, aggregated toward MCP-1 chemotaxis (14), and their presence was significantly greater in the striatum and periventricular white matter of IUGR-LPS P6 rat brains. CINC-1 is a member of the IL- 8 chemokine family, and its production by activated glial cells stimulates neutrophil recruitment. Upregulation of this chemokine is also involved in pathologic clinical conditions of brain injury, including ischemia, meningitis, acidosis, and oxidative stress $(15,16)$.
Activation of glial cells results in complex downstream events, as they serve both protective and destructive roles through the production of trophic and toxic mediators (17). Our results show a different response in glial cells when conditioned with chronic hypoxia in utero followed by inflammatory stress as compared with control. As expected, LPS resulted in reactive astrogliosis in both experimental groups, similar to that seen in previous studies $(10,18)$, and this type of pathologic change is associated with brain injury (19). However, astrogliosis is delayed in the IUGR-LPS group until P14, when it significantly increases and there is no longer a detectable difference relative to control-LPS. In LPS-treated brains from growth-restricted rat pups, there is a robust activation of microglia, which affects astrogliosis through the release of cytokines and other soluble factors. In a previous in vitro study of glial cells by Guo and Bhat (20), hypoxia and reoxygenation with LPS insult potentiated inducible nitric oxide synthase, tumor necrosis factor- $\alpha$, and NF- $\mathrm{kB}$-dependent transcription production in microglia, 

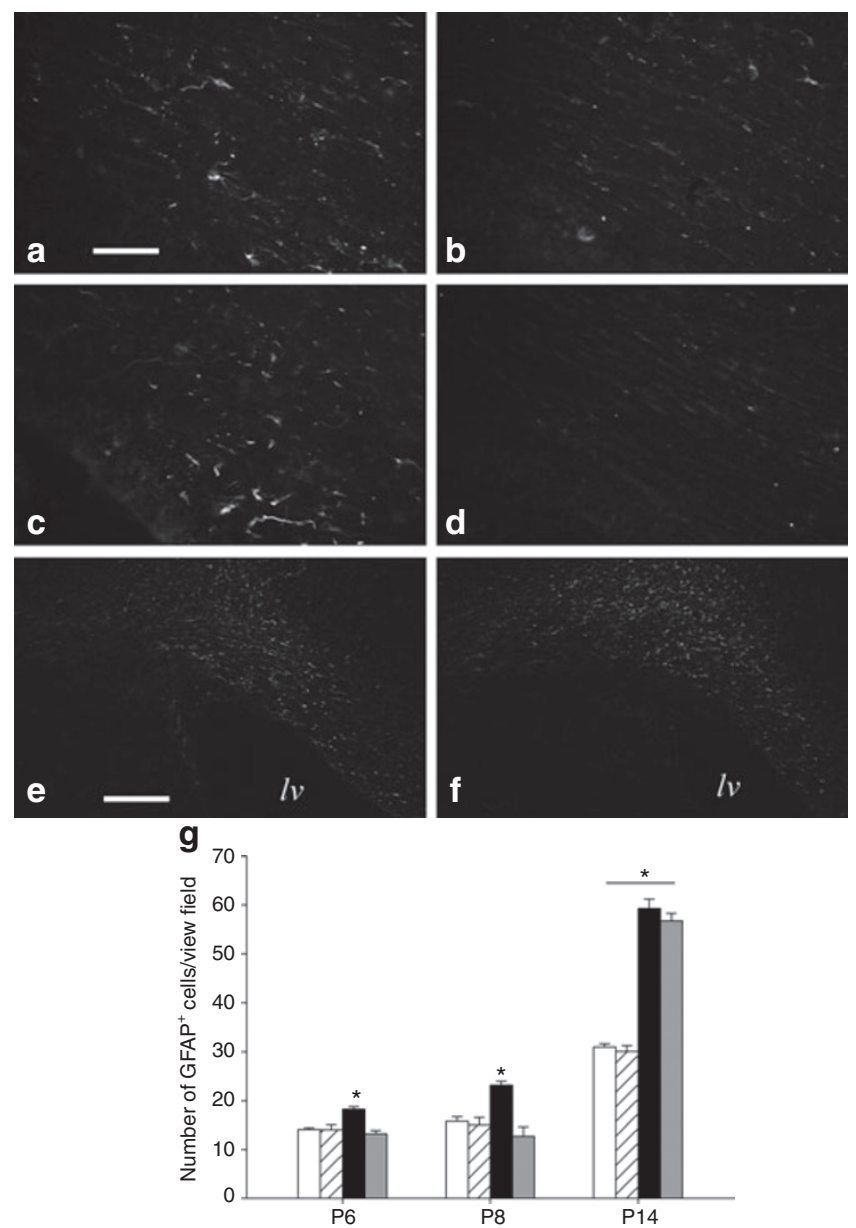

Figure 3. Delayed reactive astrogliosis in IUGR rat brain following LPS exposure. Following intracerebral LPS injection, control rat brains demonstrated progressively increased GFAP immunostaining over time (a: P6, c: P8, and $\mathbf{e : ~ P 1 4 ) , ~ a s ~ c o m p a r e d ~ w i t h ~ a ~ d e l a y e d ~ a s t r o g l i o s i s ~ i n ~ I U G R ~ r a t ~ b r a i n s ~ t h a t ~}$ was found only significantly increased at P14 (f), but not at P6 (b) or P8 (d). $\mathrm{GFAP}^{+}$cells were counted in the periventricular white matter and the data are shown in $\mathbf{g}$ (white bars: control; striped bars: IUGR; black bars: controlLPS; and gray bars: IUGR-LPS). Bar $=\mathbf{a}-\mathbf{d}: 50 \mu \mathrm{m}, \mathbf{e}, \mathbf{f :} 200 \mu \mathrm{m}$. ${ }^{*} \mathrm{P}<0.01$ control-LPS vs. control (P6 and P8); control or IUGR with LPS vs. without LPS. GFAP, glial fibrillary acidic protein; IUGR, intrauterine growth restriction; LPS, lipopolysaccharide; Iv, lateral ventricle; P, postnatal day.

which was not observed in astrocytes. However, the same combination of insults led to a substantial increase in a neuroprotective product, hemeoxygenase-1, in astrocytes but not microglia. Thus, exacerbated activation of detrimental inflammatory products in microglia and delayed astrogliosis in growth-restricted rat pups could lead to increased brain damage.

Literature supports the hypothesis that severe placental insufficiency causes brain damage, but the degree is affected by the timing, severity, and type of prenatal insult $(6,7,21,22)$. In our model of relatively moderate placental blood flow reduction, there is a lack of abnormal pathology in the brains of growth-restricted rat pups without LPS exposure. This is a strength in our study, which preconditions the neonatal rat and demonstrates the susceptibility of IUGR rat brain to secondary inflammation following LPS treatment.
IUGR rat pups demonstrated region-specific damage with an increased number of apoptotic cells primarily localized to the cortex and striatum $24 \mathrm{~h}$ following LPS. TUNEL ${ }^{+}$cells were not identified in the white matter tracts where oligodendrocytes are in abundance and injured in the pathogenesis of PVL. Knowledge of the basic anatomic regionalization of brain topography led us to believe the $\mathrm{TUNEL}^{+}$cells were potentially neurons; however, colocalization techniques to identify the apoptotic cells were inconclusive (data not shown).

Prior studies demonstrated a lowered cerebral threshold toward postnatal hypoxic stress in fetal rats subjected to placental insufficiency (21). This vulnerability was related to alterations in the pro- and antiapoptotic mechanisms, with decreased $B c l-2$ and increased $B a x$ gene expression following hypoxic insult in IUGR rat pups (23). We feel the increased apoptotic cell death in IUGR rat brains may be a result of modified apoptotic pathways and needs further investigation.

In our study, both control and IUGR rat pups demonstrated similar intensity of myelination. A secondary postnatal insult with intracerebral LPS decreased MBP stain in all experimental groups, but the hypomyelination was significantly more profound in IUGR rat pups. Clinical studies of growth-restricted infants suggest altered myelination patterns with increased prevalence of periventricular echodensities on neonatal ultrasounds and increased incidence of cerebral palsy $(5,24)$. The timing of the LPS insult at P5 correlates with a vulnerable period in human neurodevelopment and oligodendrocyte maturation when PVL onset is prevalent (25). Thus, growth restriction may sensitize immature oligodendrocytes to damage and impaired function following postnatal stress.

Ventriculomegaly is utilized in animal studies as a marker of brain injury and can result from primary developmental failure or brain cell loss (10). Clinically, increased ventricular volume correlates well with PVL and impaired cognitive and motor skills (26,27). At baseline, P14 IUGR rat pups trended toward increased ventricular dilation relative to control rat pups. Although this pattern is not statistically significant, Mallard et al. (22) demonstrated a significant size increase in the lateral ventricles of growth-restricted guinea pigs following late-gestation placental insufficiency. Similar to previous studies $(10,19)$, LPS caused pathologic ventricular dilation in all animals exposed to intracerebral LPS, but the IUGR-LPS group was most profoundly affected. Increased $\mathrm{TUNEL}^{+}$cells and a paucity of myelin immunostaining in IUGR-LPS suggest that the ventriculomegaly observed in these animals is secondary to cell loss and impaired brain structure.

In summary, we demonstrate that IUGR sensitizes the immature rat brain to produce an amplified inflammatory response after LPS insult. Glial activation and the complex cascade of cytokine and chemokine production are modified in growth-restricted rat pups, which lead to increased apoptotic cell death, impaired myelination, and extensive ventricular dilation. An adverse intrauterine environment during critical stages of growth can modify gene expression and fetal development. There are multiple studies of LPS administration that 

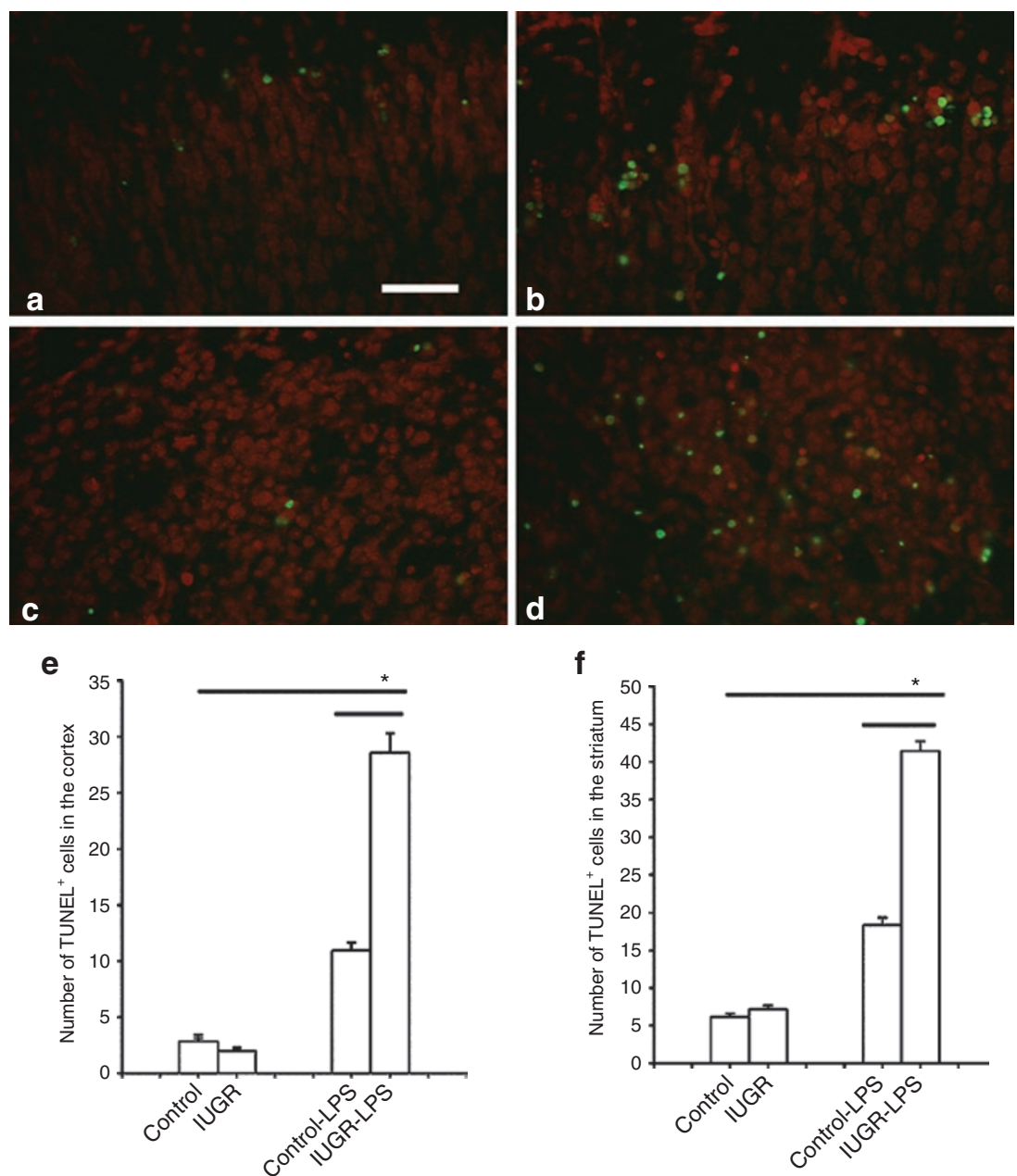

Figure 4. Cell death in the rat brain after LPS exposure. TUNEL stain (TUNEL ${ }^{+}$cells are green) with propidium iodide counterstain (red) was utilized to visualize cell death in P6 rat brain at the bregma level. There was a marked increase of TUNEL ${ }^{+}$cells in the $(\mathbf{a}, \mathbf{b})$ cortex and $(\mathbf{c}, \mathbf{d})$ striatum of both $(\mathbf{a}, \mathbf{c})$ control-LPS and (b, d) IUGR-LPS rat brains, but the increase was significantly greater in the (a) IUGR-LPS group. Quantification of cell counting data in the cortex and striatum areas is shown in $\mathbf{e}$ and $\mathbf{f}$, respectively. ${ }^{*} P<0.001$, IUGR-LPS vs. control-LPS; control or IUGR with LPS vs. without LPS. IUGR, intrauterine growth restriction; LPS, lipopolysaccharide; P, postnatal day; TUNEL, terminal deoxynucleotidyl transferase nick-end labeling.

demonstrate contrasting effects of both increased sensitization and protective preconditioning of the immature brain following hypoxic insult (28-30). However, this study is unique and investigates inflammatory effects only after exposure to prolonged hypoxia in utero.

We conclude that the immature brain in growth-restricted infants is more vulnerable to the consequences of neuroinflammation and activation of the innate immune system by LPS. The combination of chronic hypoxia and LPS-induced neuroinflammation likely works through synergistic pathways with an additive injurious effect on immature rat brains. Our model is clinically relevant, as IUGR infants are at risk for increased morbidity and mortality, including infection and neurodevelopmental handicap $(31,32)$. The differential response in IUGR rat pups relates to Barker's hypothesis of fetal programming in which an adverse environment experienced during critical developmental periods can predispose offspring to subsequent morbidities (1). Placental insufficiency creates a state of reduced oxygen availability, malnutrition, and altered endocrine status in the fetus. Delineation of how these stressors influence our results will be a focus of upcoming studies. Increased levels of CINC-1 and tissue inhibitor of metalloproteinase-1 production in growth-restricted rat pups after LPS insult lead us to hypothesize that polymorphonuclear cell recruitment and activation may be a key element in the development of observed brain damage. In addition, IUGRLPS rat brains have an increased number of TUNEL ${ }^{+}$cells, which suggests alterations in apoptotic pathways of cellular death. Future research should explore the mechanisms and temporal relationship of LPS-induced sensitization in the neonatal IUGR rat brain to provide insight into potential neuroprotective interventions.

\section{METHODS}

\section{Chemicals}

LPS (055:B5) was obtained from Sigma Chemical (St Louis, MO). Antibodies for MBP, GFAP, and ED1, and the TUNEL kit were from Millipore (Temecula, CA); the IL-1 $\beta$, MCP-1, and CINC-1 enzymelinked immunosorbent assay kits were obtained from R\&D Systems 


\section{Articles | Campbell et al.}
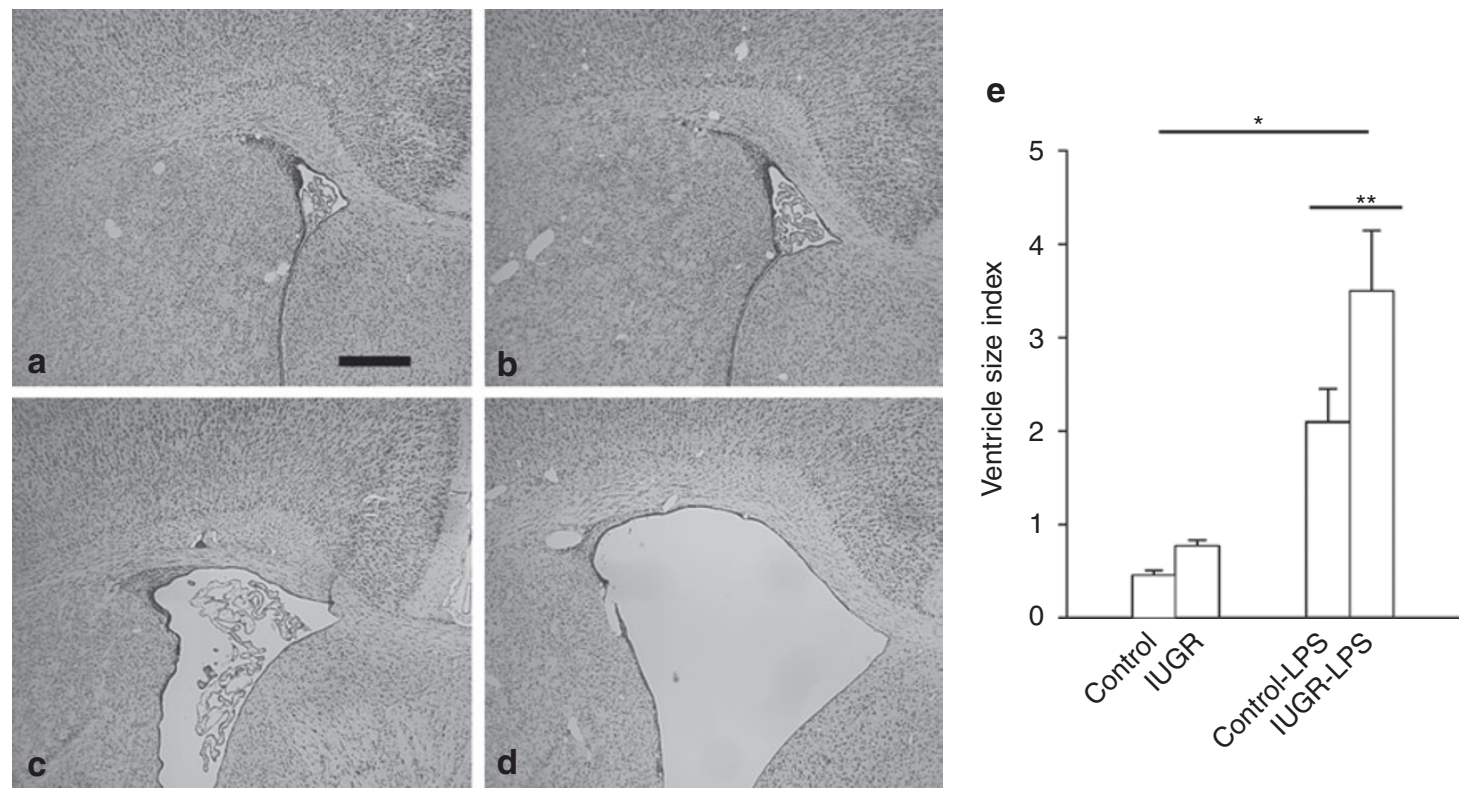

Figure 5. Changes of lateral ventricle size in rat brain after LPS injection. Hematoxylin and eosin-stained coronal sections of P14 rat brain at the bregma level demonstrated a dramatic dilation in lateral ventricle size in both control-LPS and (d) IUGR-LPS rat brains as compared with the (a) control and (b) IUGR without LPS exposure. The lateral ventricular size index at P14 is represented in e. Bar $=50 \mu \mathrm{m}$. ${ }^{*} P<0.001$ vs. control or IUGR with LPS vs. without LPS, ${ }^{* * P}<0.05$ IUGR-LPS vs. control-LPS. IUGR, intrauterine growth restriction; LPS, lipopolysaccharide; P, postnatal day.
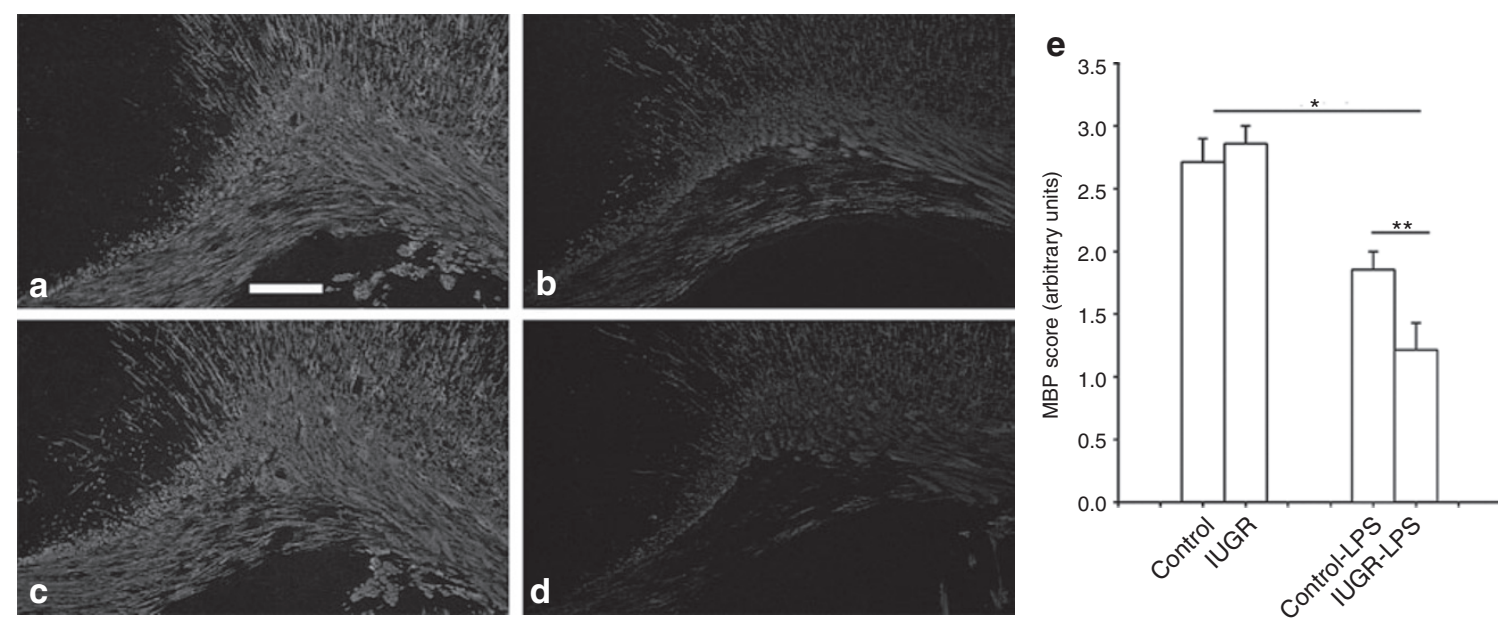

Figure 6. Detection of myelination in the rat brain by MBP immunostaining. At P14, (a) control and (b) IUGR rat brains demonstrated extensive baseline myelination of white matter that was significantly impaired by LPS treatment. However, decreased myelination (intensity and surface area) was more severe in (d) IUGR-LPS relative to (c) control-LPS. Bar $=50 \mu \mathrm{m}$. A semiquantitative method (see the Methods section) was employed to compare changes of myelination between the treatments and data are represented in e. ${ }^{*} P<0.01$, control or IUGR with LPS vs. without LPS; ${ }^{* *} P<0.05$ IUGR-LPS vs. controlLPS. IUGR, intrauterine growth restriction; LPS, lipopolysaccharide; MBP, myelin basic protein; P, postnatal day.

(Minneapolis, MN). Rat Cytokine Antibody Array 2 kit was from RayBiotech (Norcross, CA).

\section{Animal Models}

All experimental procedures performed in this study were in accordance with National Institutes of Health guidelines for the use and care of animals, with approval of all protocols by the animal care and use committee at the University of Mississippi Medical Center.

IUGR model. Timed-pregnant Sprague-Dawley rats were purchased from Harlan Sprague-Dawley (Indianapolis, IN) and housed individually. IUGR was induced by placement of a silver clip around the aorta below the renal arteries in pregnant rats at $14 \mathrm{~d}$ of gestation, as described in previously (33). Briefly, rats were anesthetized with $2 \%$ isoflurane (Butler, Edmonds, WA) in an anesthesia apparatus
(Vaporizer for Forane Anesthetic; Ohio Medical Products, Gurnee, $\mathrm{OH})$. A standardized silver clip (0.203-mm internal diameter) was placed around the isolated abdominal aorta above the iliac bifurcation, and two additional silver clips $(0.100-\mathrm{mm}$ internal diameter) were placed on both left and right branches of the uterine arteries. Sham-operated pregnant rats without clips served as controls.

All dams delivered at term, with birth weights recorded within $12 \mathrm{~h}$. Overall, mean IUGR birth weight was significantly less than controls (6.1 \pm 0.1 vs. $6.7 \pm 0.3 \mathrm{~g}$, respectively, $P<0.01$ ), with asymmetric headsparing growth as previously characterized (6). We defined IUGR as a birth weight less than the 10th percentile below the mean weight of the control rat pups born simultaneously. Control rat pups and previously defined IUGR rat pups were separately size-matched with 8 pups/dam. Growth-restricted pups with a birth weight greater than the defined 10th percentile ( $40 \%$ of total) and control rat pups with a birth weight less than this value were not utilized. 
Intracerebral injection of LPS. This study consisted of four experimental groups described as follows: control + saline, control + LPS, IUGR + saline, IUGR + LPS. Intracerebral injection of LPS was performed as previously described on P5 (refs. 9,11) Briefly, pups were anesthetized with isoflurane ( $4 \%$ induction, $1.5 \%$ maintenance) and placed in a stereotaxic apparatus with an adapter for neonatal rats (David Kopf, Tujunga, CA). The intraventricular injection was performed with a $10-\mu \mathrm{l}$ syringe using the following coordination: $1.0 \mathrm{~mm}$ posterior and $1.0 \mathrm{~mm}$ lateral to the bregma, and $3.0 \mathrm{~mm}$ deep to the skull surface. LPS $(1 \mu \mathrm{g} /$ animal) or sterile saline at a volume of $2 \mu \mathrm{l}$ was injected into the left lateral ventricle over a period of $5 \mathrm{~min}$. The intraventricular delivery was confirmed in series coronal frozen sections from the rat brains that were injected with trypan blue. For histological, immunohistochemistry, and TUNEL analysis, six animals were included in each treatment group.

\section{Cytokine Array and Enzyme-Linked Immunosorbent Assay}

Whole-brain tissue was homogenized with a glass homogenizer in $1 \mathrm{ml}$ ice-cold phosphate-buffered saline ( $\mathrm{pH} 7.2)$ with protease inhibitor. After centrifugation at $12,000 \mathrm{~g}$ for $20 \mathrm{~min}$ at $4{ }^{\circ} \mathrm{C}$, the supernatant was collected and total protein was determined by the Pierce bicinchoninic acid method. Protein concentration was adjusted to $1 \mathrm{mg} / \mathrm{ml}$. For the cytokine array, performed per manufacturer instructions, a total of four samples from the same treatment group were pooled. Signals were analyzed using ImageQuant software (Molecular Dynamics, Kent City, MI), and optical density of the target cytokine/chemokine was subtracted from the background and then expressed as a ratio to the positive control. A threshold of twofold change was considered to be significant. Enzyme-linked immunosorbent assay was performed following manufacturer's instructions. Data were acquired using a 96-well plate reader (Bio-Tek Instruments, Winooski, VT). The cytokine contents were expressed as $\mathrm{pg}$ cytokines/mg total protein.

\section{Quantification of Histology and Immunohistochemistry Data}

ED1 and GFAP immunostaining and TUNEL were quantified by cell counting. $\mathrm{ED}^{+}$and $\mathrm{GFAP}^{+}$cells were counted in the entire periventricular white matter (including corpus callosum and cingular white matter) and $\mathrm{TUNEL}^{+}$cells were counted in both the cortex and striatum. Four consecutive coronal sections at the bregma level from each animal were selected for analysis. Six digital images $(\times 10$ objective $)$ from the cortex and periventricular white matter and 12 images from the striatum, which covered most areas with positive cells, were captured for each section. The numbers of positive cells from four sections were averaged for each animal. Data are presented as the number of cells/view field.

MBP immunohistochemistry was quantified using a grading method for each slide as previously described (11): 0 , no staining; 1 , detectable weak staining; 2 , obvious staining, but $<3$ and stronger than $1 ; 3$, normal strong positive stain. Consistency and unbiased scoring was ensured by evaluation from two different people blinded to treatment; the two evaluations were then averaged.

\section{Histologic Examination, Immunohistochemistry, and TUNEL}

Fifty frozen consecutive sections $(10 \mu \mathrm{m})$ were prepared at the level of the bregma and dorsal hippocampus. Hematoxylin and eosin and Nissl staining was used to examine gross histopathology.

Immunohistochemistry was performed using a standard protocol as previously described (10). After blocking, final concentrations of primary antibodies were diluted as follows: GFAP (1:300), ED1 (1:200), and MBP (1:200). Images were captured with a charge-coupled device camera (Oly-750; Olympus, Center Valley, PA), and superimposed using Adobe Photoshop (version 7.0) software, if necessary. TUNEL staining, following the manufacturer's instructions, was used to detect cell death.

To compare the size of the lateral ventricles in relation to overall brain size, hematoxylin and eosin-stained coronal sections at the bregma level were scanned by a densitometer (Bio-Rad, Richmond, $\mathrm{CA})$ and areas $\left(\mathrm{cm}^{2}\right)$ of the left and right ventricles and whole brain were measured. Ventricular index size was calculated as the ratio of the area of each ventricle to whole brain.

\section{Statistics}

Unless otherwise indicated, results are represented as means \pm SEM. Two-way ANOVA followed by Student-Newman-Keuls test determined statistical significance among treatments, set at a $P$ value of $<0.05$.

\section{STATEMENT OF FINANCIAL SUPPORT}

This study was supported by National Institutes of Health-funded grant HL074927 and by funds from the Division of Newborn Medicine, University of Mississippi Medical Center.

\section{REFERENCES}

1. Barker DJ. In utero programming of chronic disease. Clin Sci 1998; 95:115-28.

2. Henriksen T, Clausen T. The fetal origins hypothesis: placental insufficiency and inheritance versus maternal malnutrition in well-nourished populations. Acta Obstet Gynecol Scand 2002;81:112-4.

3. Laurini R, Laurin J, Marsál K. Placental histology and fetal blood flow in intrauterine growth retardation. Acta Obstet Gynecol Scand 1994; 73:529-34.

4. Geva R, Eshel R, Leitner Y, Valevski AF, Harel S. Neuropsychological outcome of children with intrauterine growth restriction: a 9-year prospective study. Pediatrics 2006;118:91-100.

5. Jarvis S, Glinianaia SV, Torrioli MG, et al. Cerebral palsy and intrauterine growth in single births: European collaborative study. Lancet 2003; 362:1106-11

6. Rees S, Harding R, Walker D. An adverse intrauterine environment: implications for injury and altered development of the brain. Int J Dev Neurosci 2008;26:3-11.

7. Olivier P, Baud O, Bouslama M, Evrard P, Gressens P, Verney C. Moderate growth restriction: deleterious and protective effects on white matter damage. Neurobiol Dis 2007;26:253-63.

8. Volpe JJ. Neurobiology of periventricular leukomalacia in the premature infant. Pediatr Res 2001;50:553-62.

9. Cai Z, Pang Y, Lin S, Rhodes PG. Differential roles of tumor necrosis factor- $\alpha$ and interleukin- $1 \beta$ in lipopolysaccharide-induced brain injury in the neonatal rat. Brain Res 2003;975:37-47.

10. Pang Y, Cai Z, Rhodes PG. Disturbance of oligodendrocyte development, hypomyelination and white matter injury in the neonatal rat brain after intracerebral injection of lipopolysaccharide. Brain Res Dev Brain Res 2003;140:205-14.

11. Pang Y, Zheng B, Campbell LR, Fan LW, Cai Z, Rhodes PG. IGF-1 can either protect against or increase LPS-induced damage in the developing rat brain. Pediatr Res 2010;67:579-84.

12. Lee SC, Liu W, Dickson DW, Brosnan CF, Berman JW. Cytokine production by human fetal microglia and astrocytes. Differential induction by lipopolysaccharide and IL-1 $\beta$. J Immunol 1993;150:2659-67.

13. Cai Z, Pan ZL, Pang Y, Evans OB, Rhodes PG. Cytokine induction in fetal rat brains and brain injury in neonatal rats after maternal lipopolysaccharide administration. Pediatr Res 2000;47:64-72.

14. Kadhim H, Tabarki B, Verellen G, De Prez C, Rona AM, Sébire G. Inflammatory cytokines in the pathogenesis of periventricular leukomalacia. Neurology 2001;56:1278-84.

15. Yamagami S, Tamura M, Hayashi M, et al. Differential production of MCP-1 and cytokine-induced neutrophil chemoattractant in the ischemic brain after transient focal ischemia in rats. J Leukoc Biol 1999;65:744-49.

16. Katayama T, Tanaka H, Yoshida T, Uehara T, Minami M. Neuronal injury induces cytokine-induced neutrophil chemoattractant-1 (CINC-1) production in astrocytes. J Pharmacol Sci 2009;109:88-93.

17. Lehnardt S, Lachance C, Patrizi S, et al. The toll-like receptor TLR4 is necessary for lipopolysaccharide-induced oligodendrocyte injury in the CNS. J Neurosci 2002;22:2478-86.

18. Lodygensky GA, West T, Stump M, Holtzman DM, Inder TE, Neil JJ. In vivo MRI analysis of an inflammatory injury in the developing brain. Brain Behav Immun 2010;24:759-67.

19. Deguchi K, Oguchi K, Takashima S. Characteristic neuropathology of leukomalacia in extremely low birth weight infants. Pediatr Neurol 1997;16:296-300. 
20. Guo G, Bhat NR. Hypoxia/reoxygenation differentially modulates NF- $\kappa$ B activation and iNOS expression in astrocytes and microglia. Antioxid Redox Signal 2006;8:911-8.

21. Burke C, Sinclair K, Cowin G, et al. Intrauterine growth restriction due to uteroplacental vascular insufficiency leads to increased hypoxia-induced cerebral apoptosis in newborn piglets. Brain Res 2006;1098:19-25.

22. Mallard EC, Rehn A, Rees S, Tolcos M, Copolov D. Ventriculomegaly and reduced hippocampal volume following intrauterine growth-restriction: implications for the aetiology of schizophrenia. Schizophr Res 1999;40:11-21.

23. Lane RH, Ramirez RJ, Tsirka AE, et al. Uteroplacental insufficiency lowers the threshold towards hypoxia-induced cerebral apoptosis in growthretarded fetal rats. Brain Res 2001;895:186-93.

24. Padilla-Gomes NF, Enríquez G, Acosta-Rojas R, Perapoch J, HernandezAndrade E, Gratacos E. Prevalence of neonatal ultrasound brain lesions in premature infants with and without intrauterine growth restriction. Acta Paediatr 2007;96:1582-7.

25. Craig A, Ling Luo N, Beardsley DJ, et al. Quantitative analysis of perinatal rodent oligodendrocyte lineage progression and its correlation with human. Exp Neurol 2003;181:231-40.

26. Baker LL, Stevenson DK, Enzmann DR. End-stage periventricular leukomalacia: MR evaluation. Radiology 1988;168:809-15.
27. Melhem ER, Hoon AH Jr, Ferrucci JT Jr, et al. Periventricular leukomalacia: relationship between lateral ventricular volume on brain MR images and severity of cognitive and motor impairment. Radiology 2000;214:199-204.

28. Yang L, Sameshima H, Ikeda T, Ikenoue T. Lipopolysaccharide administration enhances hypoxic-ischemic brain damage in newborn rats. J Obstet Gynaecol Res 2004;30:142-7.

29. Eklind S, Mallard C, Arvidsson P, Hagberg H. Lipopolysaccharide induces both a primary and a secondary phase of sensitization in the developing rat brain. Pediatr Res 2005;58:112-6.

30. Ikeda T, Yang L, Ikenoue T, Mallard C, Hagberg H. Endotoxin-induced hypoxic-ischemic tolerance is mediated by up-regulation of corticosterone in neonatal rat. Pediatr Res 2006;59:56-60.

31. Simchen MJ, Beiner ME, Strauss-Liviathan N, et al. Neonatal outcome in growth-restricted versus appropriately grown preterm infants. Am J Perinatol 2000;17:187-92.

32. Garite TJ, Clark R, Thorp JA. Intrauterine growth restriction increases morbidity and mortality among premature neonates. Am J Obstet Gynecol 2004;191:481-7.

33. Alexander BT. Placental insufficiency leads to development of hypertension in growth-restricted offspring. Hypertension 2003;41:457-62. 